\title{
ABSTRACT STATIONARY PROCESSES
}

\author{
PARFENY P. SAWOROTNOW
}

\begin{abstract}
Concept of the multivariate stationary process is generalized to an infinite-dimensional case. Representation theorems are derived from the theory of Hilbert modules.
\end{abstract}

In this note we shall present an application of the theory of Hilbert modules to a study of multivariate stationary processes. There is a simple way to generalize the consequent theory to an infinite case (of any cardinality, in fact). Hilbert modules seem to be well suited for this purpose.

Let $G$ be a locally compact Abelian group, let $A$ be a proper $H^{*}$ algebra [1] and let $H$ be a Hilbert $A$-module [4]. In particular $G$ could be either the real line or the set of integers.

Definition. An abstract stationary (in wide sense) process is a mapping $t \rightarrow \xi(t)$ of $G$ into $H$ such that $(\xi(t+r), \xi(s+r))=(\xi(t), \xi(s))$ for all $t, r, s \in G$ (here ( , ) denotes the generalized scalar product on $H$ ). The $A$ valued function $B(t)=(\xi(t), \xi(0))=(\xi(t+s), \xi(s))$ shall be called the correlation function of $\xi(t)$.

THEOREM 1. For each continuous abstract stationary process $\xi(t)$ there exists a generalized spectral measure $P: \Delta \rightarrow P_{\Delta}$ on $\hat{G}$ and $f \in H$ such that $\xi(t)=\left(\int_{\hat{G}}(t, \alpha)-d P_{\alpha}\right)$ f for all $t \in G$ (see [6] for the definition of the generalized spectral measure). The projections $P_{\Delta}$ act on some closed submodule of $H$.

Proof. Let $H_{\xi}$ be the (closed) submodule of $H$, generated by members of $H$ of the form $\xi(t), t \in G$, i.e. $H_{\xi}$ is the closure of the set

$$
\left\{\sum_{k=1}^{n} \xi\left(t_{k}\right) a_{n} \mid t_{1}, \cdots, t_{n} \in G \text { and } a_{1}, \cdots, a_{n} \in A\right\} .
$$

For each $t \in G$ we define the operator $U_{t}$ by setting

$$
U_{t}\left(\sum_{k} \xi\left(t_{k}\right) a_{k}\right)=\sum_{k} \xi\left(t_{k}+t\right) a_{k}
$$

Presented to the Society, March 28, 1972 under the title Hilbert module valued stationary stochastic processes; received by the editors August 1, 1972 and, in revised form, November 27, 1972.

AMS (MOS) subject classifications (1970). Primary 60G10; Secondary 28A45, 46G10, 43A35, $42 \mathrm{~A} 88$.

(c) American Mathematical Society 1973 
and extending $U_{t}$ to entire $H_{\xi}$ in the obvious manner. Then each operator $U_{t}$ is $A$-linear and unitary, also $U_{t+s}=U_{t} U_{s}$ holds for all $t, s \in G$. By Theorem 3 of [5] there exists a generalized spectral measure $\Delta \rightarrow P_{\Delta}$ such that

We take $f=\xi(0) \cdots$.

$$
\xi(t)=U_{t} \xi(0)=\left(\int_{\hat{G}}(t, \alpha)^{-} d P_{\alpha}\right) \xi(0) \quad \text { for all } t \in G .
$$

Now note that the Hilbert module $H$ is also a complex Hilbert space with respect to the scalar product $\operatorname{tr}($,$) , 4$, Theorem 1]. So the theory of Masani [2] is applicable here. Thus we have:

COROLlaRY. For each continuous abstract stationary process $\xi$ there exists an $\mathrm{H}$-valued, countably additive, orthogonally scattered [2, pp. 63-64] measure $v$ on $\hat{G}$ such that $\xi(t)=\int_{\hat{G}}(t, \alpha)-d v(\alpha)$ for all $t \in G$ (the integral here is considered in the sense of pp. 75-78 of [2]).

Proof. We set $v(\Delta)=P_{\Delta} \xi(0)$. Then $v$ has the required properties (note that $P$ is also an ordinary projection-valued measure of the type considered in Example 1.5(a) of [2]). If for some $g \in H$ we define a complex valued measure $m(\Delta)=\operatorname{tr}(v(\Delta), g)$ then it will follow from Corollary 5.12 of [2] that

$$
\operatorname{tr}\left(\int_{\hat{G}}(t, \alpha)^{-} d v(\alpha), g\right)=\int_{\hat{G}}(t, \alpha) d m(\alpha)=\operatorname{tr}\left(U_{t} \xi(0), g\right) .
$$

Since $g$ was arbitrary we can conclude that $\xi(t)=U_{t} \xi(0)=\int_{\hat{G}}(t, \alpha) d v(\alpha)$ for all $t \in G$.

THeOrem 2. A necessary and sufficient condition for an A-valued function $B(t)$ to be a correlation function for some abstract stationary process is that $B(t)$ be positive definite in the sense of [5]. $B(t)$ is continuous if and only if the corresponding process is continuous.

Proof. If $B(t)$ is a positive definite $A$-function [5, p. 146] then [5, Theorem 1] there exists a unitary representation $t \rightarrow U_{t}$ of $G$ by $A$-linear operators on a Hilbert module $H$ such that $B(t)=\left(U_{t} f_{0}, f_{0}\right)$ for some $f_{0} \in H$. Define $\xi(t)=U_{t} f_{0}$; then $\xi$ is an abstract stationary process:

$$
(\xi(t+r), \xi(s+r))=\left(U_{r} U_{t} f_{0}, U_{r} U_{s} f_{0}\right)=\left(U_{t} f_{0}, U_{s} f_{0}\right)=(\xi(t), \xi(s)) .
$$

If $B$ is continuous then the representation $t \rightarrow U_{t}$ is also continuous [5, Theorem 1]. In this case $\xi(t)=U_{t} f_{0}$ is also continuous.

Conversely, let $\xi$ be an abstract stationary process and let $B(t)=$ $(\xi(t), \xi(0))$. If $t_{1}, \cdots, t_{n} \in G$ and $a_{1}, a_{2}, \cdots, a_{n} \in A$ then $f=\Sigma \xi\left(t_{k}\right) a_{k}$ is a 
member of $\boldsymbol{H}$. It follows then that

$$
\begin{aligned}
\sum_{k, j} a_{k}^{*} B\left(t_{k}-t_{j}\right) a_{j} & =\sum_{k, j} a_{k}^{*}\left(\xi\left(t_{k}\right), \xi\left(t_{j}\right)\right) a_{j} \\
& =\left(\sum_{k} \xi\left(t_{k}\right) a_{k}, \sum_{j} \xi\left(t_{j}\right) a_{j}\right)=(f, f)
\end{aligned}
$$

is positive.

We have also a generalization of Theorem 5.2 in Chapter I of [7].

TheOREM 3. Let $\mu$ be a positive $\tau(A)$-valued measure defined on Borel sets of $\hat{G}$. Then $B(t)=\int_{\hat{G}}{ }^{\circ}(t, \alpha) d \mu(\alpha)$ is a correlation function of some continuous abstract stationary process. Conversely for each continuous abstract stationary process $\xi(t)$ there exists a positive $\tau(A)$-valued Borel measure $\mu$ on $\hat{G}$ such that $(\xi(t), \xi(0))=\int_{\hat{G}}(t, \alpha) d \mu(\alpha)$ for each $t \in G$.

Proof. This is a consequence of Theorem 2 above and the generalized Bochner theorem [6].

To complete the paper we include an example of an abstract stationary process that has infinitely many components (note that multivariate stationary processes of Rozanov [7] and Wiener-Masani [8], [9] are examples of our abstract stationary processes).

ExAmple. Let $(\Omega, p)$ be a probability space and let $H$ be the set of all sequences $f=\left\{f_{n}(w)\right\}$ of random variables such that each $\left|f_{n}(w)\right|^{2}$ is summable and $\sum_{n} \int_{\Omega}|f(w)|^{2} d w<\infty((\Omega, p)$ is a measurable space with $p(\Omega)=1$ and $f$ is a sequence of measurable functions such that $\sum_{n} \int_{\Omega}\left|f\left(w^{\prime}\right)\right|^{2} d w$ is finite). Then $H$ is a Hilbert module over the algebra $A$ of infinite complex valued matrices $a=\left(a_{i j}\right)$ such that $\|a\|^{2}=\sum_{i, j}\left|a_{i j}\right|^{2}<\infty$.

Now let $\xi=\left\{\xi_{n}(t, w)\right\}_{n=1}^{\infty}$ be a sequence of mutually stationarily correlated stationary processes such that $\sum_{n} \int\left|\xi_{n}(t, w)\right|^{2} d w<\infty$ for each $t \in G$, where $G$ is either the real line or the set of all integers (if the last series diverges then we replace each $\xi_{n}$ by

$$
\left.\eta_{n}(t)=\xi_{n}(t, w) n^{-1}\left(\int_{\Omega}\left|\xi_{n}(0, w)\right|^{2} d w\right)^{-1 / 2}\right) .
$$

Then $\xi$ is an example of abstract stationary process with infinitely many components.

Let us show that the space $H$ in the above example is a Hilbert module over $A$. Let $f=\left\{f_{n}(w)\right\}$ be a member of $H$ and let $a=\left\{a_{n m}\right\} \in A$. For each $n$ consider the function $g_{n}(w)=\sum_{k} f_{k}(w) a_{k n}$. Then the inequality

$$
\left|g_{n}(w)\right|^{2} \leqq \sum_{k}\left|f_{k}(w)\right|^{2} \sum_{k}\left|a_{k n}\right|^{2}=\sum_{k}\left|f_{k}(w)\right|^{2}\|a\|^{2}
$$


shows that each $\left|g_{n}(w)\right|$ is square integrable. Also it is easy to see that

$$
\begin{aligned}
\left\{g_{n}\right\} & \in H: \sum_{n} \int\left|g_{n}(w)\right|^{2} d w \\
& \leqq \sum_{n} \int\left(\sum_{k}\left|f_{k}(w)\right|^{2} \sum_{k}\left|a_{k n}\right|^{2}\right) d w=\sum_{n}\left(\int \sum_{k}\left|f_{k}(w)\right|^{2} d w \sum_{k}\left|a_{k n}\right|^{2}\right) \\
& \leqq \sum_{k} \int\left|f_{k}(w)\right|^{2} d w \sum_{n} \sum_{k}\left|a_{k n}\right|^{2}=\sum_{k} \int\left|f_{k}(w)\right|^{2} d w\|a\|^{2} .
\end{aligned}
$$

Thus $H$ is a right module.

We define the generalized scalar product on $H$ by taking $(f, g)$ to be the matrix whose $n, m$ th entry equals to $\int\left(f_{n}(w)\right)-g_{m}(w) d w((f, g)(n, m)=$ $\left.\int\left(f_{n}(w)\right)^{-} g_{m}(w) d w\right)$. Then $(f, f) \in \tau(A)$ since $\operatorname{tr}(f, f)=\sum_{n} \int\left|f_{n}\left(w^{\prime}\right)\right|^{2} d w$ and $(f, g) \in \tau(A)$ since

$$
\begin{aligned}
4(f, g)= & (f+g, f+g)+(f-g, f-g) \\
& +i(f+i g, f+i g)-i(f-i g, f-i g) .
\end{aligned}
$$

The other axioms of a Hilbert module are easy to verify.

REMARK 1. It was pointed out to the author, after the paper was essentially finished, that a more appropriate name for the above-considered function $\xi(t)$ should be "abstract stationary random field" rather than "abstract stationary process", since $\xi(t)$ was defined on an arbitrary (commutative) topological group (see, for example, the remark in the second paragraph on p. 593 of [11]). It was also indicated by the referee that the proper title of the paper should be Abstract stationary random fields.

However, the present study was originated as an attempt to generalize multivariate processes, considered by Rozanov in his book [7], to an infinite-dimensional case. The fact that we did not restrict the domain of $\xi(t)$ to either integer or reals (discrete or continuous time) is not essential. Also M. Rosenberg uses the term "stationary processes" in his work [3]. For this and some other reasons we kept our original terminology, realizing, of course, that the present work comes under the theory of stationary random fields.

REMARK 2. In the past there have been several authors who used modules to study stationary processes. The above-considered Hilbert module is a direct generalization of modules considered by Rozanov [7] and Wiener-Masani [8], [9] (our Hilbert module can be considered as an infinite-dimensional analogue of their modules over $q \times q$ matrices).

The case of Gangolli [10] is different. He considered a particular example of a module, over the space of all bounded operators on an $L^{2-}$ space, which has an inner product assuming bounded operators as its 
values. In the present paper we consider a module over an arbitrary (proper) $H^{*}$-algebra, which may or may not be associated with HilbertSchmidt operators on an $L^{2}$-space; the inner product in our case assumes its values in the $H^{*}$-algebra involved. The module of Gangolli seems to be well suited for prediction theory. We did not go into prediction theory here. The author, however, is hopeful that our Hilbert module will prove to be useful also in doing prediction theory, at least as far as generalizing certain results of [7] and [8], [9].

\section{REFERENCES}

1. W. Ambrose, Structure theorems for a special class of Banach algebras, Trans. Amer. Math. Soc. 57 (1945), 364-386. MR 7, 126.

2. P. Masani, Orthogonally scattered measures, Advances in Math. 2 (1968), 61-117. MR 37 \#4231.

3. M. Rosenberg, Mutual subordination of multivariate stationary processes over any locally compact Abelian group, Z. Wahrscheinlichkeitstheorie und Verw. Gebiete 12 (1969), 333-343. MR 40 \#5017.

4. P. P. Saworotnow, A generalized Hilbert space, Duke Math J. 35 (1968), 191-197. MR 37 \#3333.

5. - Representation of a topological group on a Hilbert module, Duke Math. J. 37 (1970), 145-150. MR 41 \#7442.

6. - Bochner-Raikov theorem for a generalized positive definite function, Duke Math. J. 38 (1971), 117-121. MR 43 \#940.

7. Ju. A. Rozanov, Stationary random processes, Fizmatgiz, Moscow, 1963; English transl., Holden-Day, San Francisco, Calif., 1967. MR 28 \#2580; MR 35 \#4985.

8. $\mathrm{N}$. Wiener and $\mathrm{P}$. Masani, The prediction theory of multivariate stochastic processes, Acta Math. 98 (1957), 111-150. MR 20 \#4323.

9. - The prediction theory of multivariate stochastic processes. II, Acta Math. 99 (1958), 93-137. MR 20 \#4325.

10. R. Gangolli, Wide-sense stationary sequences of distributions on Hilbert space and the factorization of operator valued functions, J. Math. Mech. 12 (1963), 893-910. MR 28 \#4556.

11. A. M. Jaglom, Second-order homogeneous random fields, Proc. Fourth Berkeley Sympos. Math. Statist. and Probability, vol. II, Univ. of California Press, Berkeley, Calif., 1961, pp. 593-622. MR 26 \#4399.

Department of Mathematics, Catholic University of America, Washington, D.C. 20017 